\title{
Educação em saúde para estudantes sobre câncer de mama: relato de experiência
}

\author{
Health education for students about breast cancer: \\ experience report
}

\section{Educación de salud para estudiantes acerca del cáncer de mama: informe de experiencia}

Ana Paula Alonso Reis Mairink IFSULDEMINAS

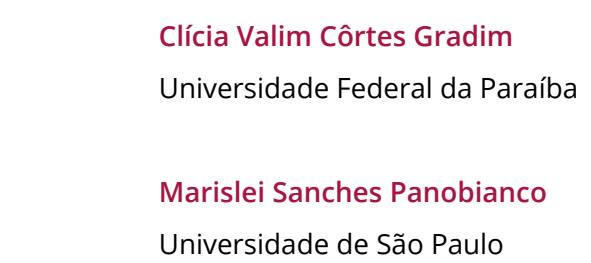

Resumo: O câncer de mama é um problema de saúde pública, sendo o segundo tipo de câncer mais comum e frequente em muIheres. O objetivo deste estudo foi relatar a experiência de preparar estudantes do curso técnico em enfermagem de um Instituto Federal de Educação, Ciência e Tecnologia para se tornarem facilitadores no processo de educação em saúde na prevenção do câncer de mama. Trata-se de relato de experiência sobre ação de educação em saúde com o tema câncer de mama. A ação de educação em saúde foi visualizada positivamente pelos estudantes, no sentido de crescimento acadêmico e profissional, pois aproximou- 
-os do assunto e possibilitou refletir sobre a importância de serem agentes promotores de saúde e de prevenção de agravos nas suas atuações profissionais. Já a comunidade, ganhou empoderamento para reconhecer precocemente alterações nas mamas, conscientização sobre a importância de hábitos e estilos saudáveis de vida e realização da mamografia.

Palavras-chave: Enfermagem. Prevenção de Doenças. Saúde Pública. Saúde da Mulher. Neoplasia Mamária.

Abstract: Breast cancer is a public health problem, being the second most common and frequent type of cancer in women. The objective is to report the experience of preparing students in the technical nursing course at a Federal Institute of Education, Science and Technology to become facilitators in the health education process for the prevention of breast cancer. This is an experience

2

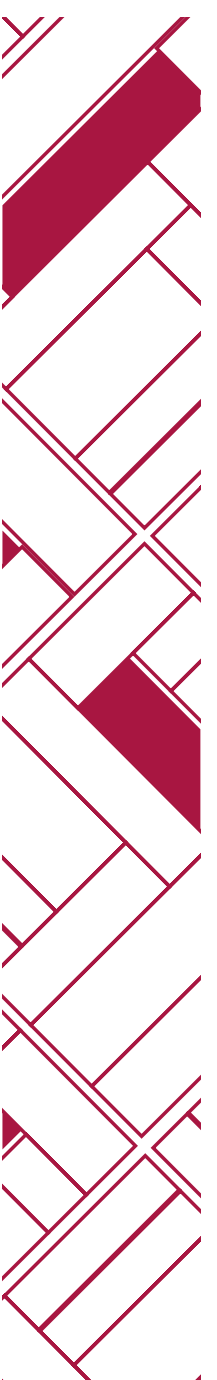
report on health education action with the theme of breast cancer. The health education action was viewed positively by students in the sense of academic and professional growth because it brought them closer to the topic and made it possible to reflect on the importance of being health promoting agents and preventing injuries in their professional activities. For the community, empowerment to recognize breast changes early, awareness of the importance of healthy habits and lifestyles, and mammography.

Keywords: Nursing. Prevention of diseases. Public health. Women's Health. Breast Neoplasm.

Resumen: El cáncer de mama es un problema de salud pública, siendo el segundo tipo de cáncer más común y frecuente en las mujeres. El objetivo es reportar la experiencia de preparar a los estudiantes del curso técnico de enfermería de un Instituto Federal de Educación, Ciencia y Tecnología para convertirse en facili- 
tadores en el proceso de educación en salud para la prevención del cáncer de mama. Este es un informe de experiencia sobre acciones de educación sanitaria con el tema del cáncer de mama. La acción de educación en salud fue valorada positivamente por los estudiantes en el sentido de crecimiento académico y profesional porque los acercó a la temática y permitió reflexionar sobre la importancia de ser agentes promotores de la salud y prevenir lesiones en sus actividades profesionales. Para la comunidad, empoderamiento para reconocer los cambios en los senos temprano, conciencia de la importancia de los hábitos y estilos de vida saludables y la mamografía.

Palabras clave: Enfermería. Prevención de enfermedades. Salud pública. La salud de la mujer. Neoplasia de mama.

Data de submissão: 19/10/2020

Data de aprovação: 14/12/2020 


\section{Introdução}

Na sociedade ocidental existe uma preocupação excessiva com o corpo. A mídia enfatiza a importância de mantê-lo esbelto, magro, com contorno e formas definidas, além de uma aparência bonita e jovial. As mamas, como partes integrantes deste corpo, nesta cultura, devem ser volumosas, salientes e atraentes, mantendo-se assim, a aparência social desejada a este órgão (OLIVEIRA; SOUSA; GARCIA, 2010).

As mamas, além de serem importantes no ato de amamentar, também possuem relevância por estarem associadas ao prazer durante o ato sexual, sendo símbolo de feminilidade e sexualidade (ROCHA et al., 2016).

Assim, a mulher que se descobre doente, com o diagnóstico de câncer de mama, pode ter crises de instabilidade emocional, medo da morte, tristeza, principalmente devido à perda da mama (advinda do tratamento cirúrgico) e queda do cabelo (advinda do tratamento quimioterápico adjuvante), o que pode levá-la a desenvolver baixa autoestima por se deparar com um corpo transformado, rosto descaracterizado pela doença e seus tratamentos, alterando sua identidade.

O câncer de mama é o segundo tipo de câncer mais comum e frequente entre as mulheres representando $24,2 \%$ do número total de casos em 2018 (WHO, 2020). A neoplasia também acomete homens, em menor proporção, 1\% do total de casos da doença (INCA, 2020).

Ainda em relação às estatísticas da neoplasia, o Instituto $\mathrm{Na}$ cional de Câncer (INCA) estimou cerca de 66.280 casos novos da doença para o ano de 2020. Em relação à mortalidade, um quantitativo de óbitos de 17.763, sendo 17.572 de mulheres e 189 de homens, de acordo com dados do ano de 2018 do Sistema de Informação de Mortalidade (INCA, 2020). 
A neoplasia mamária não possui causa única e sabe-se que a idade é um dos fatores de risco mais importantes para a enfermidade pois, em torno de quatro a cada cinco casos da doença, acontecem em pessoas após os 50 anos (INCA, 2020). Entretanto, apesar de o aparecimento da patologia em mulheres jovens, com idade inferior a 40 anos, ser menos frequente, este merece destaque, pois nesse público jovem a doença possui índice mais elevado de mortalidade (38\%), enquanto que nas idosas compreende cerca de 33\% (ALMEIDA et al., 2015) devido as características clínicas, imuno-histoquímicas e patológicas do tumor serem mais agressivas, estádio mais avançado, maior diâmetro do tumor e chances de metástase (PEREIRA; VIPIANA; SILVA, 2017).

Os riscos para o desenvolvimento da doença estão relacionados a fatores ambientais e comportamentais (obesidade e sobrepeso após a menopausa, consumo de bebida alcoólica, exposição frequente a radiações ionizantes (raios-X) (INCA, 2020), fatores da história reprodutiva e hormonal (não ter tido filhos, primeira menstruação antes de 12 anos, parar de menstruar (menopausa) após os 55 anos, primeira gravidez após os 30 anos e reposição hormonal pós-menopausa, principalmente por mais de cinco anos) e os fatores genéticos e hereditários (casos de câncer de mama na família, principalmente antes dos 50 anos, história familiar de câncer de ovário, história familiar de câncer de mama em homens, alteração genética, especialmente nos genes BRCA1 e BRCA2) (INCA, 2020) sendo esses fatores de risco não modificáveis mas passíveis de prevenção por meio da detecção precoce das alterações na mama e da realização de mamografia de rotina.

Nesse sentido de prevenção dos fatores de risco, a Atenção Primária à Saúde (APS) constitui-se na principal porta de entrada da comunidade no Sistema Único de Saúde (SUS), tornando-se assim um espaço importante para trabalhar com educação em saú- 
de no sentido de fortalecer as ações em saúde no âmbito individual ou coletivo, como o câncer de mama.

De acordo com as diretrizes para a detecção precoce do câncer de mama no Brasil (INCA, 2015) é necessário trabalhar com a prevenção primária que é o controle dos fatores de risco reconhecidos, os citados anteriormente (modificáveis e não modificáveis) e também trabalhar a prevenção secundária que é a detecção precoce das alterações na mama através do rastreamento (estratégias de conscientização da comunidade para identificar mudanças habituais das mamas em momentos diferentes do ciclo de vida e a divulgação dos principais sinais e sintomas da neoplasia mamária e mamografia).

Dados do INCA mostram que cerca de $30 \%$ dos casos de neoplasia mamária poderiam ser evitados mediante a adoção de hábitos mais saudáveis, tais como a prática de atividade física, alimentação saudável, peso adequado, evitar bebidas alcoólicas e o uso de hormônios sintéticos (anticoncepcionais e terapia de reposição hormonal) e amamentar (INCA, 2020).

Os sinais e sintomas mais comuns da doença são o aparecimento de pequenos nódulos nas axilas ou no pescoço, saída espontânea de líquido anormal pelos mamilos, nódulo (caroço) fixo e geralmente indolor, pele da mama avermelhada, retraída ou parecida com casca de laranja, alterações no bico do peito (mamilo) (INCA, 2020).

Nos Estados Unidos, na década de 50, surgiu a estratégia do autoexame das mamas no intuito de reduzir o diagnóstico de tumores em estádios avançados. Ao fim da década de 90, ensaios clínicos apontaram que o autoexame das mamas não contribuía para reduzir a mortalidade pela doença. Assim, a partir daí, vários países começaram a adotar a estratégia breast awareness, que incentiva estar alerta para a saúde das mamas (THORNTON; PILLARISETTI, 2008). 
No Brasil, as diretrizes para a detecção precoce do câncer de mama vão ao encontro do que já estava sendo adotado por diversos países e contraindicam o rastreamento por meio do autoexame das mamas, devido à pouca procura por parte das mulheres para a confirmação diagnóstica e pelo elevado percentual de exames falso-negativos (INCA, 2015).

O rastreamento é de suma importância para o diagnóstico precoce da neoplasia e primordial para diminuir a mortalidade. Assim, as diretrizes atuais (INCA, 2015) indicam que a mamografia de rotina deve ser solicitada às mulheres na faixa etária de 50 a 69 anos e pode ser feita a cada dois anos. O exame de mamografia é o único que, aplicado em programas de rastreamento, possui eficácia comprovada de diminuição da mortalidade por neoplasia mamária (THORNTON; PILLARISETTI, 2008).

Nesse sentido, o diagnóstico precoce aumenta as taxas de cura, sobrevida da doença e diminui as repercussões que a neoplasia pode ocasionar no corpo e na vida da mulher, fazendo-se necessárias campanhas de conscientização na comunidade para o reconhecimento precoce de alterações na mama, adoção de hábitos e estilo de vida saudáveis (prevenção primária), reconhecimento de alterações nas mamas e a importância do exame de rastreamento (mamografia rotina) na faixa etária recomendada pelo Ministério da Saúde e INCA (prevenção secundária). É importante esclarecer que o INCA é um órgão que auxilia o Ministério da Saúde no desenvolvimento e na coordenação das ações de prevenção e controle de cânceres no Brasil.

A adoção da estratégia de diagnóstico precoce colabora para a redução do estádio do tumor e, por vezes, é conhecida como down-staging (WHO, 2007). Essa estratégia aponta para a importância da educação dos profissionais de saúde, e da mulher, para 
reconhecer sinais e sintomas do câncer de mama, além do acesso facilitado e rápido aos serviços de saúde (INCA, 2018).

Nesse contexto, a educação em saúde pode colaborar para transformar cenários pois a utilização de ferramentas educativas possibilita tornar mais acessível e popular a importância dos cuidados em saúde, prevenção de doenças e tratamento de agravos. Nessa linha de pensamento, merecem destaque as ações efetivas de entidades de saúde ou grupos, sem o auxílio governamental de promover saúde da população e o desempenho dos grupos multiprofissionais pois, a educação em saúde, é realizada através da ação educativa em saúde. Assim, a educação em saúde é um meio de tornar popular cuidados de saúde, métodos de prevenção e tratamento de agravos, tornando a linguagem médica mais acessível (SILVA; PELAZZA, SOUZA, 2016).

No contexto da formação de estudantes da saúde, em especial daqueles inseridos na educação profissional técnica de nível médio na área da enfermagem, conhecidos como cursos técnicos em enfermagem, a educação em saúde se faz um instrumento de trabalho importante, especialmente quando é utilizada como meio de se problematizar a realidade pois, o intercâmbio entre o saber profissional e do senso comum é um benefício para a conscientização e construção de pessoas mais críticas e bem informadas, proporcionando ainda, um vínculo com a comunidade envolvida na ação (CERVERA; PARREIRA; GOULART, 2011).

Ainda no sentido de troca de saberes entre a instituição de ensino e a comunidade, a área da saúde tem, nas atividades de extensão, a oportunidade dessa aproximação com vistas a uma atuação transformadora. Entende-se por extensão o processo cultural, educativo e científico que possibilita a articulação entre pesquisa e ensino de maneira indissociável, viabilizando a relação transformadora entre a universidade e a sociedade. Assim, essa 
indissociabilidade entre ensino-pesquisa-extensão está vinculada ao processo de geração de conhecimento e formação de pessoas, e tem o estudante, como o protagonista de sua formação técnica, importante para sua realidade profissional (CORRÊA, 2006).

O objetivo do presente estudo é relatar a experiência de preparar estudantes do curso técnico em enfermagem de um Instituto Federal de Educação, Ciência e Tecnologia para se tornarem facilitadores no processo de educação em saúde para a prevenção do câncer de mama.

\section{Material e Métodos}

Este estudo configura-se num relato de experiência sobre uma ação de educação em saúde, envolvendo estudantes, professores e comunidade, em relação à temática câncer de mama e, nesse sentido, obtém dispensa em relação à apreciação do Comitê de Ética em Pesquisa da instituição responsável pelo projeto.

Entende-se por relato de experiência uma ferramenta da pesquisa descritiva que possibilita a reflexão sobre uma atividade, ação ou um conjunto delas e que contribui para uma abordagem de situações vivenciadas no âmbito profissional, de interesse da comunidade científica (FIGUEIREDO, 2004).

As atividades preparatórias para a ação de educação em saúde aconteceram na disciplina de Assistência Materno-Infantil I e II com os estudantes do segundo módulo do curso Técnico em Enfermagem no ano de 2018.

A proposta educativa contemplou a confecção de materiais teóricos e didáticos, que foram os banners, folders e fotos das ações realizadas. Foi utilizada também, durante a ação de educa- 
ção em saúde, a mama didática (com nódulos) existente no laboratório de enfermagem da instituição de ensino.

Além disso, os estudantes realizaram em um diário de campo notas de observação, que são as descrições da audição e observação no campo de pesquisa (STRAUSS; CORBIN, 2008) e que nos serviu de apoio na elaboração desse relato de experiência.

Para o cumprimento das etapas descritas acima, os estudantes se dividiram em grupos, conforme afinidade de cada um com o trabalho que seria executado por cada grupo:

Grupo 1 - responsável pelo levantamento de materiais e informações sobre a temática câncer de mama;

Grupo 2 - digitalização do conteúdo realizado pelo grupo 1, cotação e impressão de materiais teóricos e didáticos para utilizar na ação de educação em saúde;

Grupo 3 - elaboração da arte do folder e do banner;

Grupo 4 - apresentação do material à comunidade nos ambulatórios municipais, anotação no diário de campo das observações, registro da ação de educação em saúde com foto.

Em cada grupo foi eleito um responsável, pelos próprios estudantes que o compuseram, no intuito de que este responsável fizesse a mediação entre os demais membros do seu grupo, otimizando e conduzindo a organização das tarefas. Todas as etapas e os grupos de trabalho foram supervisionados pela professora responsável pela disciplina.

Os estudantes do primeiro e terceiro módulos do curso Técnico em Enfermagem também foram incluídos na ação de educação em saúde. Os do primeiro módulo, contribuíram confeccionando laços e arrecadando bexigas rosas, que foram usados e distribuídos durante as orientações; e os do terceiro módulo, nas orientações propriamente ditas durante o estágio curricular supervisionado do curso, visto que tinham conhecimento do assunto por 
terem percorrido o segundo módulo do curso e tido a disciplina de Assistência Materno Infantil I e II.

O período de preparação da ação de educação em saúde teve duração de aproximadamente três meses, com reuniões e debates com os estudantes do primeiro, segundo e terceiro módulos do curso e a professora da disciplina de Assistência Materno-Infantil I e II, que nessa ocasião, cabe salientar, era também a coordenadora do curso, o que facilitou o envolvimento dos estudantes dos módulos I e III na ação de educação em saúde.

As orientações sobre a temática se deram de forma expositiva dialogada e foram realizadas pelos estudantes durante a semana de 01 a 05 de outubro de 2018, num evento de extensão denominado “Outubro Rosa 2018: conscientização da importância da prevenção do câncer de mama". Teve duração média de 4 horas, em cada um dos dias que a mesma aconteceu, além do tempo destinado a preparação do material pelos estudantes, sendo que a duração total das ações foi de 40 horas (preparação e execução da proposta educativa).

O público-alvo foi a comunidade interna de uma instituição da rede federal de ensino (orientados pelos estudantes do módulo III, durante o estágio curricular supervisionado) e a comunidade local do município em que essa instituição de ensino está situada (orientados pelos estudantes do módulo II), ou seja, os usuários que aguardavam por atendimento médico em dois ambulatórios municipais. Participaram da ação de educação em saúde cerca de 100 pessoas.

As orientações foram supervisionadas por professores do curso que atuavam em campo de estágio (orientações para a comunidade interna), e pela professora da disciplina de Assistência Materno-Infantil I e II (orientações para a comunidade local).

Durante a ação de educação em saúde, os participantes foram orientados/estimulados a formularem perguntas e fazerem 
questionamentos sobre o que era apresentado, assim como exporem suas experiências e outras dúvidas pertinentes ao assunto, utilizando-se assim, do método participativo. Este método, além de possibilitar o oferecimento de informações acerca do câncer de mama à comunidade participante, também permitiu o acolhimento, livre comunicação, questionamentos sobre o assunto em pauta, o que contribui para uma melhor aprendizagem dos envolvidos (STRECK, 2016).

\section{Resultados e Discussão}

Os resultados da ação de educação em saúde para os estudantes e comunidade local são oriundos das notas de observação (descrições da audição e observação no campo de pesquisa) realizadas pelos estudantes do segundo módulo do curso, no diário de campo (caderno de anotações).

A orientação realizada pelos estudantes abordou temática importante para a comunidade, em especial, o público feminino. A utilização dos materiais preparados por eles (banner e folder), e a mama didática (com nódulo), contribuíram para uma melhor apreensão do assunto pela comunidade, visto a dinamicidade dos recursos utilizados, corroborando com estudo realizado (O'MAHONY et al., 2017).

Para os estudantes, a comunidade que recebeu as orientações, adquiriu consciência sobre a necessidade de adotar e manter hábitos e estilos saudáveis de vida (prevenção primária), necessidade de cuidados com a mama, atentando para reconhecer alterações que levam à suspeita de modificações significativas, e a suma relevância da realização do exame de mamografia na faixa etária recomendada (prevenção secundária), esses dois últimos 
quesitos sendo apontados, como também importantes, em estudo que contemplou reflexões de estratégias de diagnóstico precoce (MIGOWSKI et al., 2018) e indo ao encontro das diretrizes atuais para detecção do câncer de mama no Brasil, recomendadas pelo Ministério da Saúde e INCA (INCA, 2015).

A detecção precoce é um meio efetivo de redução da mortalidade pela neoplasia mamária (MIGOWSKI et al., 2018) e fica evidente que a atividade física e a alimentação influenciam no bem-estar e, também, na qualidade de vida do indivíduo e são importantes no processo de preservação e promoção da saúde (MUNHOZ et al., 2016).

A ação de educação em saúde foi considerada pelos estudantes como uma oportunidade de crescimento acadêmico e profissional, além de se sentirem-se atuantes na questão da promoção de saúde e prevenção de doença, em especial, acerca desta neoplasia.

Dentro do contexto educacional, a proposta da ação de educação em saúde proporcionou a integração e envolvimento de estudantes e professores do curso Técnico em Enfermagem e contribuiu para a experiência da humanização e do trabalho em equipe, ferramentas importantes para o efetivo cuidado em saúde.

A humanização do atendimento em saúde é essencial à população, uma vez que é compreendida como acolhimento e parte dos princípios de integralidade da assistência e equidade, proporcionando o envolvimento do usuário, além de promover a criação de espaços que valorizem a dignidade do profissional e dos próprios usuários dos serviços (PASTANA, 2019).

O trabalho em equipe, na área da saúde, compreende partiIhar o planejamento, dividir tarefas, cooperar, colaborar e interagir de modo democrático, agregando os diversos atores, práticas, saberes, necessidades e interesses (LOPES, 2019). Ele surge da necessidade do estabelecimento de metas e objetivos em comum, num 
plano de trabalho bem definido, que possibilite o desenvolvimento do crescimento individual e do grupo, com o cuidado centrado no cliente e comunidade envolvidos (SILVEIRA; SENA; OLIVEIRA, 2011), contribuindo para a promoção de saúde e prevenção de agravos.

Assim, essa proposta educativa contemplou esses quesitos importantes do trabalho em equipe e proporcionou aos estudantes uma vivência prática, ainda na instituição de ensino, do quanto é importante eles trabalharem em equipe nos seus ambientes de trabalho futuros.

\section{Considerações Finais}

A ação de educação em saúde proporcionou aos estudantes 14 melhor conhecimento acerca da temática proposta, humanização em saúde e trabalho em equipe, maior facilidade para serem agentes promotores de saúde e contribuírem, no âmbito profissional, para a promoção de saúde e prevenção de agravos.

Quanto à comunidade que recebeu as orientações, houve maior conscientização acerca da importância da adoção de hábitos e estilos de vida saudáveis, reconhecimento de alterações mamárias e necessidade da realização de mamografia, como maneiras de prevenção e contribuição para a detecção precoce da neoplasia mamária.

Trata-se, portanto, de um exemplo de atividade que deve ser replicada, e sempre aprimorada, considerando-se o impacto positivo entre os estudantes e comunidade, corroborando com as diretrizes propostas pelo Ministério da Saúde e INCA de estratégias no intuito de detecção precoce dessa neoplasia (rastreamento e diagnóstico precoce). 
Educação em Saúde para Estudantes sobre Câncer de Mama... Ana Paula Alonso Reis Mairink • Clícia Valim Côrtes Gradim, et al...

\section{Referências}

ALMEIDA, T.G. et AL. ViVÊNCIA DA MULHER JOVEM COM CÂNCER DE MAMA E mastectomizada. Revista de Enfermagem, v.19, n.3, 2015. Disponível em: HTTPS://WWW.SCIELO.BR/PDF/EAN/V19N3/1414-8145-EAN-19-03-0432.PDF. ACESSO EM: 30 Ago.2020.

CERVERA, D.P.P.; PARREIRA, B.D.M.; GOULART, B.F. EDUCAÇÃO EM SaÚde: PeRCepÇão dos enfermeiros da ATEnÇÃo básicA em Uberaba-MG. Ciênc. \& Saúde Coletiva, Rio de Janeiro, v.16, N.11, P.1547-1554, 2011. DisPONÍVEL EM: HTTPS://WWW.SCIELO.BR/PDF/CSC/V16s1/A90v16s1.PDF. ACESSO EM: 25 SET.2020.

CORRÊA, E.J. et al. A Área dA SAÚde e a Implementação da Política NaCional de Extensão. Rev. Bras. Extensão Universitária, v.4, N.2, p.7-13, 2006. DISPONIVEL EM: HTTPS://PERIODICOS.UFFS.EDU.BR/INDEX.PHP/RBEU/ARTICLE/ VIEW/956. ACESSO EM: 04 Ago.2020.

Figueiredo, N.M.A. Método e Metodologia na Pesquisa Científica. EDITORA: DifusÃo, 2004, 247F.

INSTITUTO NACIONAL DO CÂNCER JOSÉ ALENCAR GOMES DA SiLVA. INCA. [DATA desconhecida]. Tipos de CÂncer: CÂncer de mama. [MODIFICAdO 2020 AgOSTO 21]. Disponível EM: HTTPS://WWW.INCA.GOV.BR/TIPOS-DE-CANCER/CANCER-DE-MAMA. ACESSO em 25 Set.2020.

INSTITUTO NACIONAL DO CÂNCER JOSÉ ALENCAR GOMES DA SILVA. INCA. [DATA desconhecida]. DetecçÃo PReCoce do CÂnCer de mama. [MODIFICADO 2018 NOVEMBRO 13]. DISPONIVEL EM: HTTPS://WWW.INCA.GOV.BR/ EN/NODE/1208. ACESSO EM: 25 SET.2020.

INSTITUTO NACIONAL DE CÂNCER JOSÉ ALENCAR GOMES DA SILVA. INCA. DiRetrizes PARA A DETECÇÃo PRECOCE do CÂNCER DE MAMA No 
Educação em Saúde para Estudantes sobre Câncer de Mama... Ana Paula Alonso Reis Mairink • Clícia Valim Côrtes Gradim, et al...

Brasil/Instituto Nacional de Câncer José Alencar Gomes da Silva - Rio DE JANEIRO: INCA, 2015. 168P.

LOPES, M.T.S.R. Educação Permanente e Humanização na transformação das práticas na Atenção Básica. Rev Min Enferm, v.23, e-1161, p.1-7, 2019. DISPONÍVEL EM: HTTPS://PESQUISA.BVSALUD.ORG/BRASIL/RESOURCE/PT/BIBLIO-1005032. ACESSO EM: 25 AGO.2020.

MIGOWSKI, A. et Al. Diretrizes PARA Detecção PRECoCe do CÂNCER DE MAMA NO BRASIL. II - NOVAS RECOMENDAÇÕES NACIONAIS, PRINCIPAIS EVIDÊNCIAS E CONtrovérsias. Cad. Saúde Pública, v.34, n.6, 2018. Disponível em: https:// DOI.ORG/10.1590/0102-311X00074817

MUNHOZ, M.P. ET AL. EFEITO dO EXERCíCIO FísICO E DA NUTRIÇÃO NA PREVENÇÃO do CÂncer. Revista Odontológica de Araçatuba, v.37, n.2, P.09-16, 2016. DisPONÍVEL EM: HTTPS://APCDARACATUBA.COM.BR/REVISTA/2016/08/TRABALHO5.PDF. ACESSO EM: 25 SET.2020.

OLIVEIRA, C.L.; SOUSA, F.P.A.; GARCIA, C.L. CÂnCER E IMAGEM CORPORAL: Perda da identidade feminina. Rev. Rene, Fortaleza, 11 (número especial), P.53-60, 2010. DISPONIVEL EM: HTTP://WWW.PERIODICOS.UFC.BR/INDEX.PHP/ RENE/ARTICLE/VIEW/4659. ACESSO EM: 25 JUN.2020.

O'MAHONY, M. ET AL. INTERVENTIONS FOR RAISING BREAST CANCER AWARENESS IN women. Cochrane Database Syst Rev, v.2: CD011396, 2017. Disponível EM: HTTPS://WWW.SCIELOSP.ORG/ARTICLE/CSP/2018.v34N6/E00074817/. ACESSO EM: 09 AGO.2020.

PEREIRA, H.F.B.E.S.A.; VIAPIANA, O.S.; SILVA, K.L.T. Aspectos ClínIcos e Patológicos do Câncer de Mama em Mulheres Jovens Atendidas na FCecon entre 2003 e 2013. Revista Brasileira de Cancerologia, v.63, N., P.103-109, 2017. DISPONÍVEL EM: HTTPS://RBC.INCA.GOV.BR/REVISTA/INDEX.PHP/ REVISTA/ARTICLE/VIEW/145/82. ACESSO EM: 26 JUN.2020. 
Educação em Saúde para Estudantes sobre Câncer de Mama... Ana Paula Alonso Reis Mairink • Clícia Valim Côrtes Gradim, et al...

PASTANA, I.C.A.S.S. Práticas Humanizadoras na Atenção Básica: uma REVISÃO SISTEMÁTICA QUALITATIVA. SínTESE DE EVIDÊNCIAS QUALITATIVAS PARA INFORMAR POLÍTICAS DE SAÚDE, 2019; DisPONÍVEL EM: hTTP://DOCS.BVSALUD. ORG/BIBLIOREF/2019/10/1022200/BIS-V20N2-SINTESE-DE-EVIDENCIAS-QUALITATIVAS-54-62.PDF. ACESSO EM: 10 AGO.2020.

ROCHA, J.F.D. et Al. Mastectomia: as CICATRIZES NA SEXUAlidAde FEMININA. Rev enferm UFPe online, ReCife, 10(suPl.5), P.4255-4263, 2016. DisPONIVEL EM: HTTPS://PERIODICOS.UFPE.BR/REVISTAS/REVISTAENFERMAGEM/ARTICLE/ VIEWFILE/11171/12705. ACESSO EM: 26 JUN.2020.

SILVA, M.I.; PELAZZA, B.B.; SOUZA, J.H. EduCAÇÃo E SAÚdE: RELATO dE EXPERIÊNCIAS DE AÇõES EDUCATIVAS PARA SAÚDE EM COMUNIDADES SOCIALMENTE VULneráveis. Diversa Prática, v.3, N.1, P.17-40, $1^{\circ}$ Semestre, 2016. Disponível EM: HTTP://WWW.SEER.UFU.BR/INDEX.PHP/DIVERSAPRATICA/ARTICLE/VIEW/49615. ACESSO EM: 08 AGo.2020.

STRAUSS, A.; CORBIN, J. TÉCNICAS E PROCEDIMENTOS PARA O deSENVOlVImento de Teoria Fundamentada. 2. ed. Porto Alegre: Artmed, 2008.

STRECK, D.R. Metodologias PARTICIPATIVAS DE PESQUiSA E EDUCAÇÃo POPULAR: Reflexões sobre critérios de qualidade. Comunicação Saúde Educação, V.20, N.58, P.537-547, 2016. DisPONÍVEL EM: HTTPS://WWW.SCIELOSP.ORG/ARTICLE/ICSE/2016.v20N58/537-547/PT/. ACESSO EM: 09 AGO.2020.

SILVEIRA, M.R.; SENA, R.R.; OLIVEIRA, S.R. O PROCESSO DE TRABALHO DAS EQUIPES DE SAÚdE DA FAMÍlIA: IMPLICAÇÕES PARA A PROMOÇão DA SAÚde. Rev Min Enferm., V.15, N.22, P.196-201, 2011. DisPONÍVEL EM: HTTP://WWW.REME. ORG.BR/ARTIGO/DETALHES/25. ACESSO EM: 10 AGO.2020.

THORNTON, H.; PILLARISETTI, R.R. 'BREAST AWARENESS' AND 'BREAST

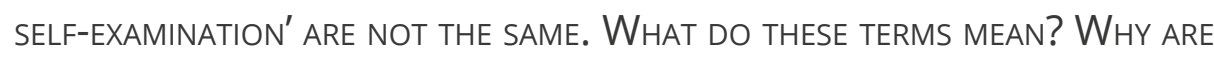
they confused? What can we do? European Journal of Cancer, Oxford, 
Educação em Saúde para Estudantes sobre Câncer de Mama... Ana Paula Alonso Reis Mairink • Clícia Valim Côrtes Gradim, et al...

V.44, N.15, P.2118-2121, 2008. DisPONíVEL EM: HTTPS://WWW.SCIENCEDIRECT.COM/SCIENCE/ARTICLE/ABS/PII/S0959804908006643. ACESSO EM: 25 SET.2020.

WORLD HEALTH ORGANIZATION (WHO). INTERNATIONAL AGENCY FOR RESEARCH ON CANCER. GLOBOCAN, 2020. DisPonível EM: HTTPS://WWW.INCA.GOV.BR/CONTROLE-DO-CANCER-DE-MAMA/CONCEITO-E-MAGNITUDE. ACESSO EM: 25 JUN.2020.

WORLD heAlth ORGANizATiON (WHO). Early Detection. Geneva, 2007. (CANCER CONTROL: KNOWLEDGE INTO ACTION: WHO GUIDE FOR EFFECTIVE PROGRAMMES). DISPONÍVEL EM: HTTPS://APPS.WHO.INT/IRIS/BITSTREAM/ HANDLE/10665/43743/9241547338_ENG.PDF;'SEQUENCE=1.. ACESSO EM: 07 AGO.2020.

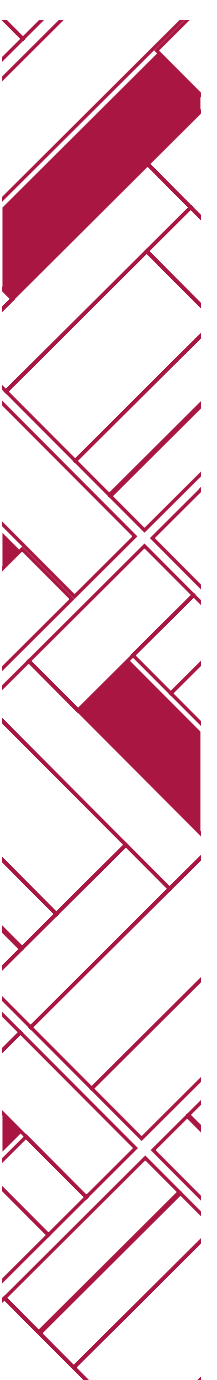

